\title{
Computed tomography and magnetic resonance imaging features of solid serous cystadenomas of the pancreas
}

\author{
JIE-YU CHEN, HAI-YAN CHEN, YAO PAN, DAN SHI and RI-SHENG YU \\ Department of Radiology, The Second Affiliated Hospital of Zhejiang University School of Medicine, \\ Hangzhou, Zhejiang 310009, P.R. China
}

Received September 13, 2018; Accepted March 20, 2019

DOI: $10.3892 / \mathrm{ol} .2019 .10346$

\begin{abstract}
Solid serous cystadenomas (SSC) of the pancreas are the rarest benign type of serous cystic neoplasms and are frequently misdiagnosed, resulting in unnecessary invasive surgical procedures. A retrospective study was performed to collect imaging features of SSC using computed tomography $(\mathrm{CT})$ and magnetic resonance imaging. The clinical data and imaging study of 5 female patients with pathologically confirmed SSC (mean age, 44.2 years; range, 23-69 years) diagnosed between January 2006 and July 2017 were retrospectively analyzed. The imaging study was assessed by two experienced radiologists in consensus. All patients had tumors with a mean size of $2.3 \mathrm{~cm}$ (range, $1.5-3.2 \mathrm{~cm}$; $4 / 5$ lesions $\leq 3 \mathrm{~cm}$ ). The tumors were located in the tail of pancreas in 3 cases and in the neck of the pancreas in 2 cases. The tumors were all well defined, with an ovoid and lobulated shape and fibrous capsules. Consequently, they presented as low density on unenhanced CT images, marked hyperintensity on T2-weighted images in 4 cases and slight hyperintensity in 1 case. However, they exhibited heterogeneous wash-in and wash-out enhancement in 2 cases and moderate and prolonged enhancement in 3 cases. The apparent diffusion coefficient (ADC) values of the lesions were $>2 \times 10^{-3} \mathrm{~mm}^{2} / \mathrm{sec}$. Therefore, a small tumor size, signal intensity on T2-weighted images, high ADC values, fibrous capsules and enhancement patterns may be indicative imaging features of SSCs.
\end{abstract}

Correspondence to: Dr Ri-Sheng Yu, Department of Radiology, The Second Affiliated Hospital of Zhejiang University School of Medicine, 88 Jiefang Road, Hangzhou, Zhejiang 310009, P.R. China E-mail: risheng-yu@zju.edu.cn

Abbreviations: SSC, solid serous cystadenoma; CT, computed tomography; MR, magnetic resonance; $\mathrm{PCN}$, pancreatic cystic neoplasm; SCN, serous cystic neoplasm; MRCP, magnetic resonance cholangiopancreatography; DWI, diffusion weighted images; ADC, apparent diffusion coefficient; pNET, pancreatic neuroendocrine tumor; SPN, solid pseudopaillary neoplasm of pancreas; BD-IPMN, branch duct intraductal papillary mucinous neoplasm

Key words: pancreas, solid serous cystadenoma, computed tomography, magnetic resonance imaging

\section{Introduction}

Reporting of pancreatic cystic neoplasms (PCNs) have increased in the past decade due to the widespread use and rapid improvement of abdominal cross-sectional imaging technologies (1), as well as an increased awareness of their existence. PCNs are a heterogeneous group of tumors with serous cystic neoplasms (SCNs) being one of the most common (1). SCNs account for $\sim 1-2 \%$ of pancreatic exocrine tumors $(2,3)$, amounting to $\sim 20 \%$ of all cystic tumors of the pancreas (4). SCNs have four morphologic patterns: Microcystic (honeycomb), macrocystic (oligocystic), mixed (polycystic) and solid types (5). Unlike the first three types, the solid type is composed of small acini with glandular spaces (6) which cannot be observed under a microscope. Solid type tumors present as a mass on radiological images with overlapping features, such as the presence of enhancement, resemble those of other solid tumors $(3,4)$. Solid serous cystadenomas (SSCs) of the pancreas were first described by Perez-Ordonez et al (7) and are reported to be the rarest type of SCNs $(2,3,8)$, accounting for only $3 \%$ (5). Correct preoperative diagnosis is required as the management of the conditions differs considerably. SSCs are benign and are managed conservatively (6). Surgery is only recommended for symptoms related to the compression of adjacent organs (9) or an uncertain diagnosis following complete workup (10). Solid tumors require surgery if they are symptomatic, malignant or display potential malignancy. The aim of the current study was to analyze the radiological results of patients with SSCs affirmed by pathology and to summarize the significant features to assist future clinical diagnosis and interventions.

\section{Materials and methods}

Subjects. A total of 132 patients with pathologically confirmed SCNs that had undergone surgical treatment at The Zhejiang University School of Medicine, Second Affiliated Hospital (Hangzhou, China) between January 2006 and July 2017 were reviewed and 5 of these patients with SSCs (determined by pathological analysis) and high-quality radiological images were retrospectively reviewed. The present study was approved by the Institutional Review Board of Zhejiang University School of Medicine, Second Affiliated Hospital. All patients were female, with a mean age of 44.2 years 
(range, 23-69 years). They had no previous medical history of pancreatitis or pancreatic neoplasm. Of these, 3 patients were incidentally observed to present with a pancreatic mass without discomfort, 1 patient had upper left abdominal pain and the other had abdominal distension and nausea. A total of 4 patients presented with normal pancreatic tumor markers, whereas 1 patient exhibited a slightly elevated carbohydrate antigen 19-9 levels (47.5 IU/ml; normal <37 IU/ml). The time interval between the first scan and surgery was 3-40 days. All patients underwent surgical resection and were without any evidence of recurrence during follow-up. The clinical features of the 5 patients are summarized in Table I.

Radiological examination. The preoperative computed tomography and magnetic resonance (MR) images of all the patients were available. In addition, 4 patients underwent magnetic resonance cholangiopancreatography (MRCP). CT scanning was performed by multidetector-row helical CT scanners (Somatom Definition AS; Siemens Healthineers, Erlangen, Germany). Unenhanced images of the upper abdomen were initially obtained using a collimation of $5 \mathrm{~mm}$ with pitch $1.2 \mathrm{~mm}$, tube voltage of $120 \mathrm{kV}$ and a tube current of $200 \mathrm{~mA}$. Depending on the patient's body weight, $80-100 \mathrm{ml}$ of contrast medium (Omnipaque 300 mg/ml; GE Healthcare, Chicago, IL, USA) was administered intravenously at an injection rate of 3.5-4.0 ml/sec. A three-phase contrast study was performed with $1.25 \mathrm{~mm}$ collimation through the pancreas while patients held their breath. Images were obtained at the arterial, portal venous and equilibrium phases (25, 60 and $100 \mathrm{sec}$ following injection, respectively).

MR scanning was performed using a GEDiscovery MR7503 Tesla MRI scanner (GE Healthcare). Fat-saturated T1-weighted images, T2-weighted images and additional contrast-enhanced T1-weighted images were obtained in all patients in the transverse section without breathing artifact. The contrast medium (Omniscan, $0.1 \mathrm{ml} / \mathrm{kg}$ body weight; GE Healthcare) was injected at a rate of $2 \mathrm{ml} / \mathrm{sec}$. Gadolinium-enhanced study on T1-weighted images was first performed at $20 \mathrm{sec}$ following the initiation of intravenous contrast administration. Multiphasic images were obtained in the portal phase at 45-52 sec, in the equilibrium phase at 75-82 sec and in the delayed phase at 135-142 sec (slice thickness, $4 \mathrm{~mm}$; interslice gaps, $4 \mathrm{~mm}$ ). Diffusion-weighted images (DWI) were also acquired in all patients using the following parameters: Repetition time/echo time, 6,000/53.0 msec; thickness, $5 \mathrm{~mm}$; matrix size, 128x128; $\mathrm{b}$ value $=0$ and $800 \mathrm{sec} / \mathrm{mm}^{2}$. The apparent diffusion coefficient (ADC) maps were generated on the operating console. The MRCP examinations were performed on a 1.5 Tesla MRI scanner (GE Healthcare). The following imaging sequences were acquired: Axial T2 fast spin echo with fat saturation [repetition time (TR) 6,000/echo time (TE) 63-86] with $7 \mathrm{~mm}$ slices and coronal (TR 3,333.3/TE 617-705) reconstructed with $1.8 \mathrm{~mm}$ slices.

Image analysis. A total of 2 experienced abdominal radiologists with no prior knowledge of the pathological outcome separately reviewed the clinical information and imaging data, and a consensus was reached. The following morphological features were evaluated: i) Location of the lesion (head, neck, body or tail of pancreas); ii) size of the lesion (maximal diameter of the tumor); iii) lesion contour (round, ovoid, lobulated or irregular); iv) margin of the lesion (well- or ill-defined); v) appearance of the lesion (the density on CT, the signal intensity on MRI and the enhancement pattern), vi) presence of a capsule; vii) central scar formation; viii) calcification; ix) hemorrhage; $x$ ) pancreatic ductal dilatation; $x i$ ) invasion of adjacent tissues; xii) enlarged lymph nodes; and xiii) distant metastases.

In terms of appearance characteristics, the density of the lesion was recorded, compared with the surrounding pancreatic parenchyma and labeled as hyper-, iso- or hypo-attenuating. The signal intensity of lesions on the unenhanced T1-weighted images was compared with that of the surrounding pancreas and graded as hypo-, iso- and hyper-intense. The signal intensity of lesions on T2-weighted images contrasting with that of the surrounding pancreas and spleen was described as 'very hyper-, hyper-, iso- and hypo-intense' when the signal intensity of the lesion was higher than that of the spleen, not higher than that of the spleen but higher than that of the pancreas, similar to that of the pancreas and lower than that of the pancreas, respectively (11). The dynamic enhancement pattern was evaluated in the homogeneity of the tumor (homogeneous or heterogeneous). The regions of interest (ROIs) placed on the lesions with the largest diameter on the ADC maps in order to cover the entire length of the lesion were defined. The final ADC values were expressed as the mean value $\mathrm{mm}^{2} / \mathrm{s}$ of the ROIs of three selected slices. The presence of scars and fibrous capsules appeared as increasing, weak and/or prolonged enhancement centrally and peripherally, respectively. Hemorrhage was identified by the existent areas of hyperintensity on T1-weighted images and the corresponding hypointensity on T2-weighted images.

Pathologic analysis. A total of 2 senior pathologists investigated the gross and microscopic specimens and reached an agreement. Macroscopically, the 5 resected specimens revealed a solid and well-circumscribed appearance. Histologically, hematoxylin and eosin staining revealed that the encapsulated tumors consisted of cuboidal or polygonal cells and dense collagenous septa. The tumor cells had small round nuclei and clear or pale eosinophilic cytoplasm, without cytological atypia or abnormal mitotic figures. Neoplastic cells were sometimes arranged in small acini. Microscopically, the surgical margins were negative with no vascular or nerve invasion.

\section{Results}

CT and MR images revealed 3 masses in the tail of pancreas and 2 in the neck. The mean tumor size among the 5 patients was $2.3 \mathrm{~cm}$ (range, $1.5-3.2 \mathrm{~cm}$; $4 / 5$ of lesions $\leq 3 \mathrm{~cm}$ ). All cases had radiological evidence of well-circumscribed masses, which were ovoid $(n=3)$ and lobulated $(n=2)$. A total of 4 cases demonstrated low-density solid masses (Fig. 1) on unenhanced CT and a high signal intensity on T2-weighted images and isointensity on DWI (Fig. 2). Only 1 case revealed a slight hypodense mass with central low density protruding from the pancreatic tail on unenhanced CT and was heterogeneously hyperintense on T2-weighted images and slightly hyperintense on DWI. On unenhanced T1-weighted images, 5 cases revealed homogeneous and low intensity lesions. 
Table I. Clinical aspects in the 5 cases.

\begin{tabular}{|c|c|c|c|c|c|c|c|}
\hline Case & $\begin{array}{l}\text { Age } \\
\text { (years) }\end{array}$ & Sex & Symptom(s) & $\begin{array}{c}\text { CA19-9 } \\
\text { level (IU/ml) }\end{array}$ & $\begin{array}{c}\text { Preoperative } \\
\text { diagnosis }\end{array}$ & $\begin{array}{l}\text { Surgical } \\
\text { procedure }\end{array}$ & Outcome \\
\hline 1 & 69 & Female & Incidental diagnosis & Normal & pNET & $\begin{array}{l}\text { PPP with } \\
\text { cholecystectomy }\end{array}$ & Uneventful \\
\hline 2 & 27 & Female & Incidental diagnosis & Normal & SPN & LDP & Uneventful \\
\hline 3 & 49 & Female & $\begin{array}{l}\text { Left upper } \\
\text { abdominal pain }\end{array}$ & Normal & $\begin{array}{l}\text { Pancreatic } \\
\text { cystadenpoma }\end{array}$ & $\begin{array}{l}\text { LDP with } \\
\text { splenectomy }\end{array}$ & Uneventful \\
\hline 4 & 53 & Female & Incidental diagnosis & Normal & $\begin{array}{l}\text { Pancreatic } \\
\text { adenocarcinoma }\end{array}$ & PPP & Uneventful \\
\hline 5 & 23 & Female & $\begin{array}{l}\text { Abdominal distension, } \\
\text { nausea }\end{array}$ & 47.5 & SPN & PPP & Uneventful \\
\hline
\end{tabular}

Normal CA19-9 levels were considered as <37 IU/ml. CA19-9, carbohydrate antigen 19-9; pNET, pancreatic neuroendocrine tumor; SPN, solid pseudopapillary neoplasm of pancreas; PPP, partial pancreatectomy and pancreaticojejunostomy; LDP, laparoscopic distal pancreatectomy.
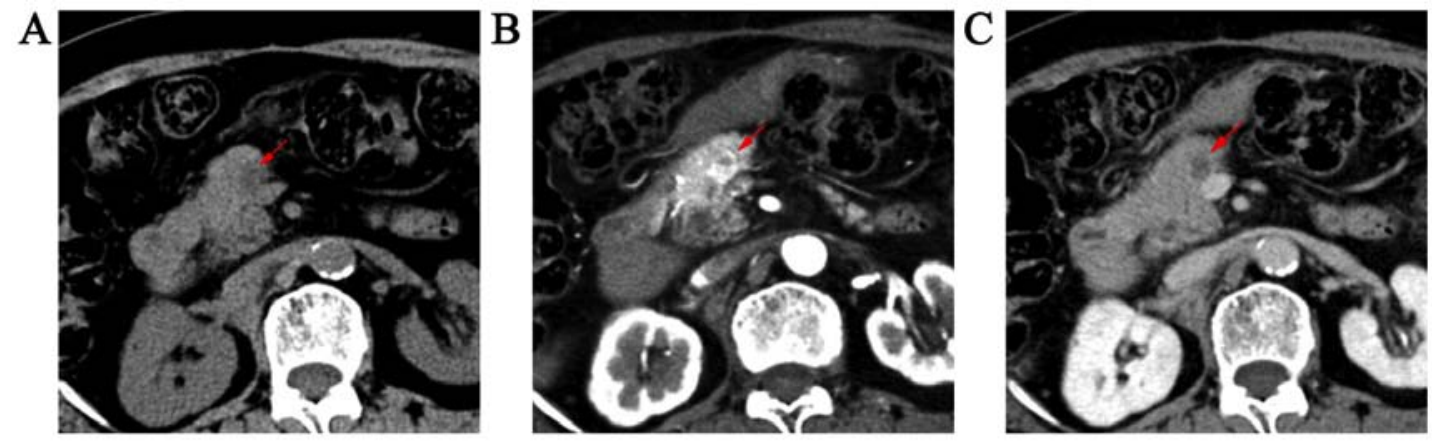

Figure 1. A 69-year-old female with a solid serous cystadenoma in the neck of pancreas. (A) Precontrast scan revealed a homogeneous and low-density lesion (arrow) in the pancreatic neck. (B) Scan obtained during arterial phase revealed a strong enhanced lesion (arrow) with increasingly enhanced capsule and a weakly enhanced zone as a result of a high concentration of collagenous stroma. (C) Scan obtained during the equilibrium phase revealed a low-density lesion (arrow).

The 5 masses revealed two different enhancement patterns on enhanced CT and MR imaging. Of these, 2 masses exhibited strong and heterogeneous enhancement with a poorly enhanced zone in the arterial phase, washout and had a homogenous appearance, hypodensity or isodensity during portal venous and equilibrium phases (Figs. $1 \mathrm{~B}$ and $\mathrm{C}$ and 3). Moderate and gradual enhancement in the arterial phase, and prolonged and faintly spotted enhancement during the portal venous and equilibrium phases were observed in three masses. The three masses were hypo-dense/intense relative to the surrounding normal pancreatic tissue in all enhanced phases. Additionally, increased enhancement of the periphery of the lesions was confirmed on the 3 phases in 4 cases, while an area of strong enhancement surrounded by a dark thin rim was exhibited in 1 patient, which revealed the presence of a capsule. The enhanced manifestations of lesions on MR imaging were similar to those of the CT images.

The ADC values of the 5 lesions were $2.46 \times 10^{-3}, 2.18 \times 10^{-3}$, $2.13 \times 10^{-3}, 2.21 \times 10^{-3}$ and $2.53 \times 10^{-3} \mathrm{~mm}^{2} / \mathrm{sec}$, respectively. MRCP revealed increased intensity in the four lesions (Fig. 4), almost equivalent to that of common bile duct and distal ductal dilation $(5.5 \mathrm{~mm})$ in 1 case. No intratumoral calcification, central scar, hemorrhage, enlarged lymph nodes, adjacent invasion or distant metastasis were observed in all five patients. The imaging features are summarized in Table II. All cases were misdiagnosed.

\section{Discussion}

Owing to the widespread use and rapid improvement of cross-sectional imaging studies, there has been an increase in the detection of PCNs (1). SSCs are benign entities and may be misdiagnosed as pancreatic neuroendocrine tumors (pNET), solid pseudopapillary neoplasms of the pancreas (SPN), ductal adenocarcinomas and metastatic carcinomas (12). Therefore, distinguishing between SSCs and malignant types allows the selection of an appropriate treatment strategy. Previous studies on SSCs presenting as solid and enhanced masses are limited, with the majority being case reports. Additionally, the radiological features of the masses are sporadically described. Jais et al (10) conducted an international and multicenter study involving 2,622 SCNs and reported that SSCs type accounted for 5\% of these cases. Thus, the aim of the current study was to analyze the radiological results of patients with SSCs and to summarize the significant features to improve the diagnostic accuracy.

SSCs were initially described in 1996 as solid pancreatic tumors composed of cells that are morphologically and 

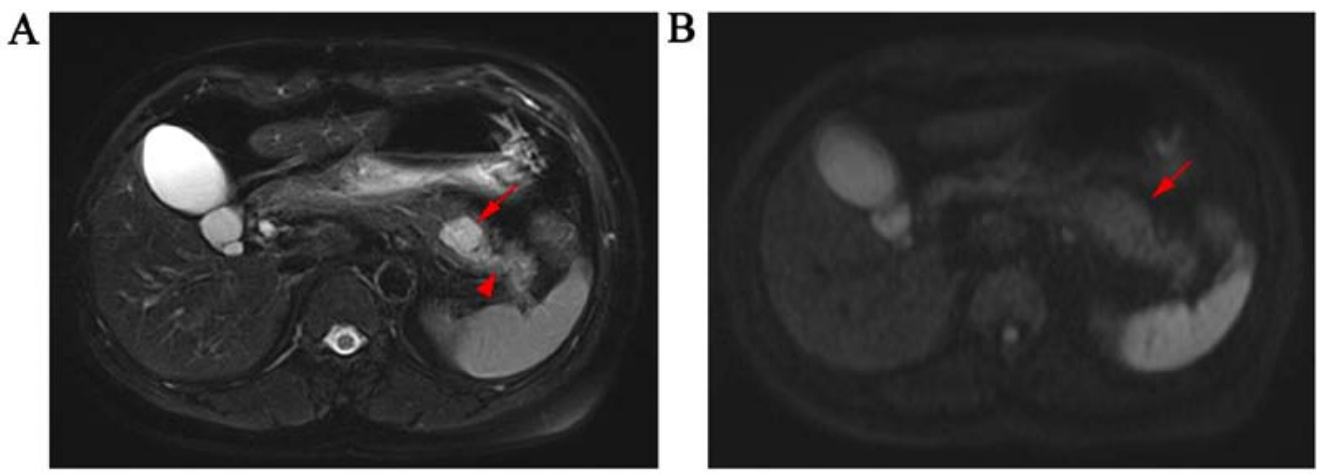

Figure 2. A 53-year-old female with a solid serous cystadenoma located in the tail of pancreas (arrow), misdiagnosed as pancreatic adenocarcinoma. (A) T2-weighted image revealed a higher intensity than the spleen (arrow) and a distal ductal dilation (arrowhead). (B) Diffusion weighted images revealed an isointense mass (arrow).
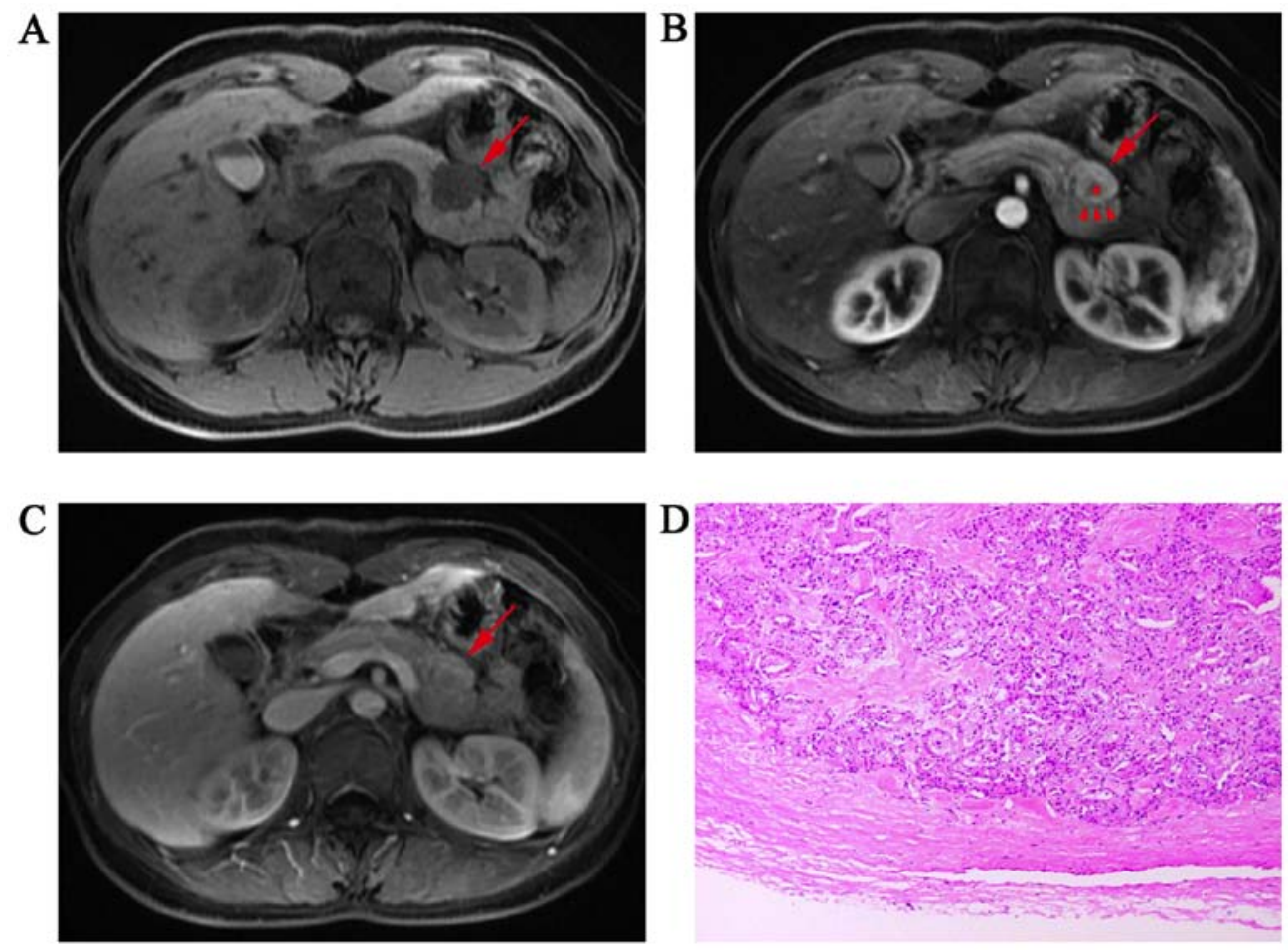

Figure 3. A 27-year-old female with a solid serous cystadenoma in the tail of pancreas. (A) Axial T1-weighted image revealed a homogeneously and significantly hypointense tumor (arrow) in the body/tail of pancreas. (B) Post-contrast T1-weighted image obtained during arterial phase revealed a marked enhancement of the tumor (arrow) with a poorly enhanced intratumoral zone (asterisk) and hypointense capsule (arrowhead). (C) Scan obtained during portal phase revealed homogeneously prolonged enhancement (arrow). (D) Representative figure of an encapsulated tumor composed of cells arranged in small acini, separated by a thick, hypocellular fibrous band. Magnification x100.

histochemically indistinguishable from those of SCNs (7). Subsequent case reports supported SSCs as an SCN subtype $(8,13)$. Previous studies reported that SSCs consist of serous cystadenoma cells that do not secrete fluid $(6,14)$ or they comprise a large number of small cysts $(3,15,16)$ measuring tenths of a millimeter (13).

To the best of the authors' knowledge, only 22 SSCs have been previously reported in detail, and the clinical and radiologic characteristics of these cases are presented in Table III $(2,3,7,8,12-26)$. The demographic characteristics of the 22 cases were as follows: i) Male and female ratio, 1:1.6; ii) mean age, 60.6 years; iii) $~ 50 \%$ of lesions were located in the body of the pancreas $(9 / 22,45.5 \%)$; iv) the mean size of the lesions was $2.8 \mathrm{~cm}$; and v) $15 / 22$ of the lesions (68.2\%) were $\leq 3 \mathrm{~cm}$ in diameter. SSCs were located in different parts of the pancreas and occurred most frequently in females in their sixth decade. Consistent with previously published studies, all the SSCs occurred in adult females in the present study, although the patients involved in the present study were younger. The mean size of lesions was $2.3 \mathrm{~cm}$, which was smaller than that reported in the aforementioned studies. Of the five cases in the present study, four of them had biomarker expression levels (carcinoembryonic antigen, $\alpha$-fetoprotein, CA19-9 and CA21-5 within the expected range; data not shown) within the normal range, and this was similar to previously published studies $(3,8,16,18,19,21,22,23,25)$. 
Table II. Summary of the imaging features in the five cases.

\begin{tabular}{|c|c|c|c|c|c|}
\hline \multirow[b]{2}{*}{ Case } & \multirow[b]{2}{*}{ Location } & \multirow[b]{2}{*}{ Size $(\mathrm{cm})$} & \multicolumn{2}{|c|}{ Radiological findings } & \multirow{2}{*}{$\begin{array}{l}\text { Apparent diffusion } \\
\text { coefficient }\left(\mathrm{mm}^{2} / \mathrm{sec}\right)\end{array}$} \\
\hline & & & Computed tomography & Magnetic resonance & \\
\hline 1 & Neck & 1.5 & $\begin{array}{l}\text { Well-defined and hypodense, } \\
\text { wash-in and wash-out enhancement } \\
\text { with poor enhanced zone, } \\
\text { enhanced capsule }\end{array}$ & $\begin{array}{l}\text { Low signal on T1WI, very } \\
\text { high signal on T2WI, } \\
\text { isosignal on DWI, wash-in } \\
\text { and wash-out enhancement } \\
\text { with weakly enhanced zone, } \\
\text { enhanced capsule }\end{array}$ & $2.46 \times 10^{-3}$ \\
\hline 2 & Tail & 3.0 & $\begin{array}{l}\text { Well-defined and slight hypodense, } \\
\text { wash-in and wash-out enhancement } \\
\text { with poor enhanced zone and capsule }\end{array}$ & $\begin{array}{l}\text { Low signal on T1WI, high } \\
\text { signal on T2WI, slight high } \\
\text { signal on DWI, wash-in and } \\
\text { wash-out enhancement with } \\
\text { poor enhanced zone and capsule }\end{array}$ & $2.18 \times 10^{-3}$ \\
\hline 3 & Tail & 2.0 & $\begin{array}{l}\text { Well-defined and hypodense, } \\
\text { Lobulated change, moderate and } \\
\text { prolonged enhancement, } \\
\text { enhanced capsule }\end{array}$ & $\begin{array}{l}\text { Low signal on T1WI, very } \\
\text { high signal on T2WI, iso-signal } \\
\text { on DWI, moderate and prolonged } \\
\text { enhancement, enhanced capsule }\end{array}$ & $2.13 \times 10^{-3}$ \\
\hline 4 & Tail & 1.8 & $\begin{array}{l}\text { Well-defined and hypodense, } \\
\text { moderate and prolonged enhancement } \\
\text { with obviously enhanced capsule }\end{array}$ & $\begin{array}{l}\text { Low signal on T1WI, very high } \\
\text { signal on T2WI, isosignal on } \\
\text { DWI, moderate and prolonged } \\
\text { enhancement, enhanced capsule }\end{array}$ & $2.21 \times 10^{-3}$ \\
\hline 5 & Neck & 3.2 & $\begin{array}{l}\text { Well-defined and hypodense, lobulated } \\
\text { change, moderate and prolonged } \\
\text { enhancement, enhanced capsule }\end{array}$ & $\begin{array}{l}\text { Low signal on T1WI, very high } \\
\text { signal on T2WI, isosignal on DWI, } \\
\text { moderate and prolonged } \\
\text { enhancement, enhanced capsule }\end{array}$ & $2.53 \times 10^{-3}$ \\
\hline
\end{tabular}

T1WI, T1-weighted imaging; T2WI, T2-weighted imaging; DWI, diffusion weighted images.
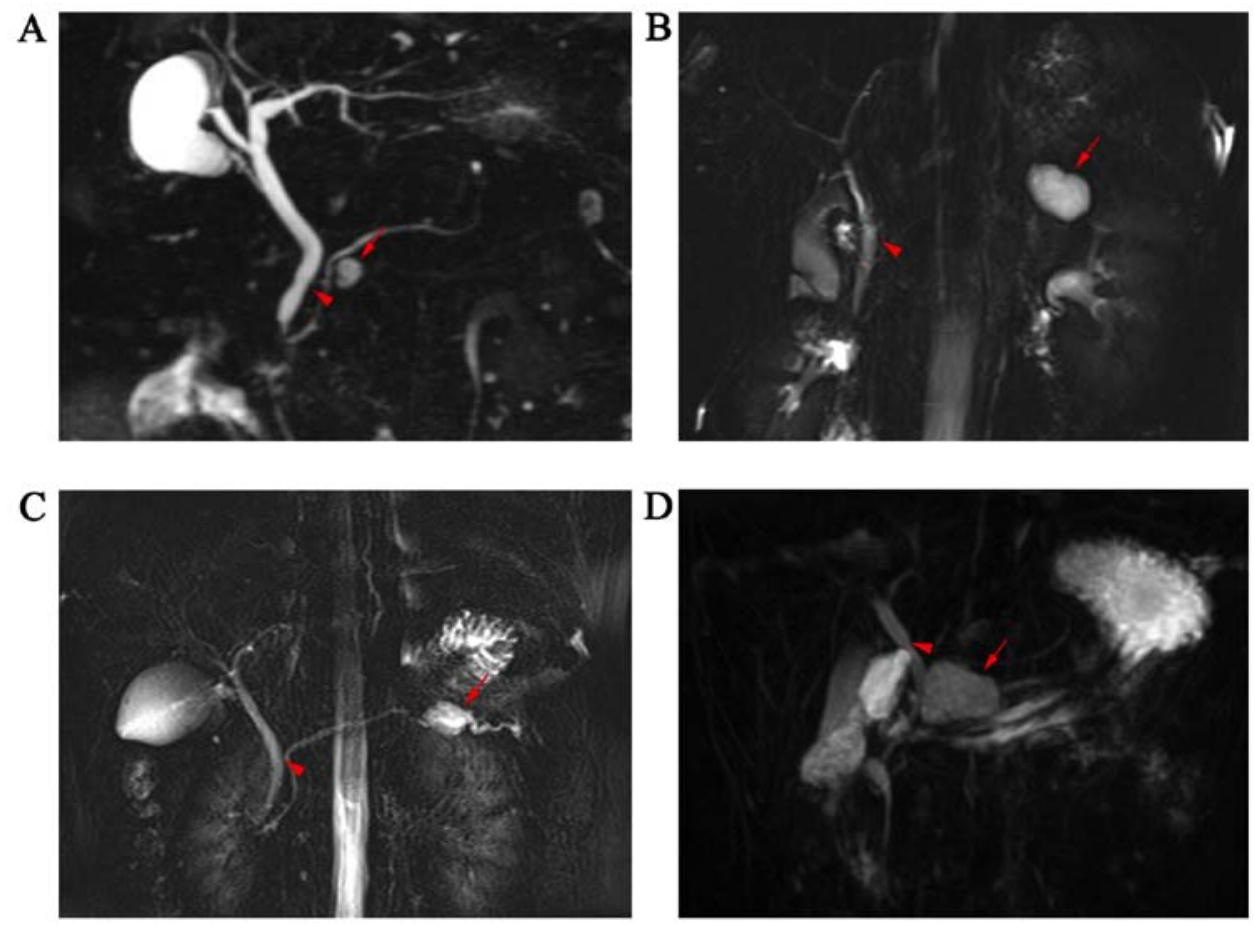

Figure 4. Lesions of solid serous cystadenoma of the pancreas in four of the patients. (A-D) Magnetic resonance cholangiopancreatography revealed a very high signal intensity of the pancreatic masses (arrow), equal to that of their common bile ducts (arrowhead). 


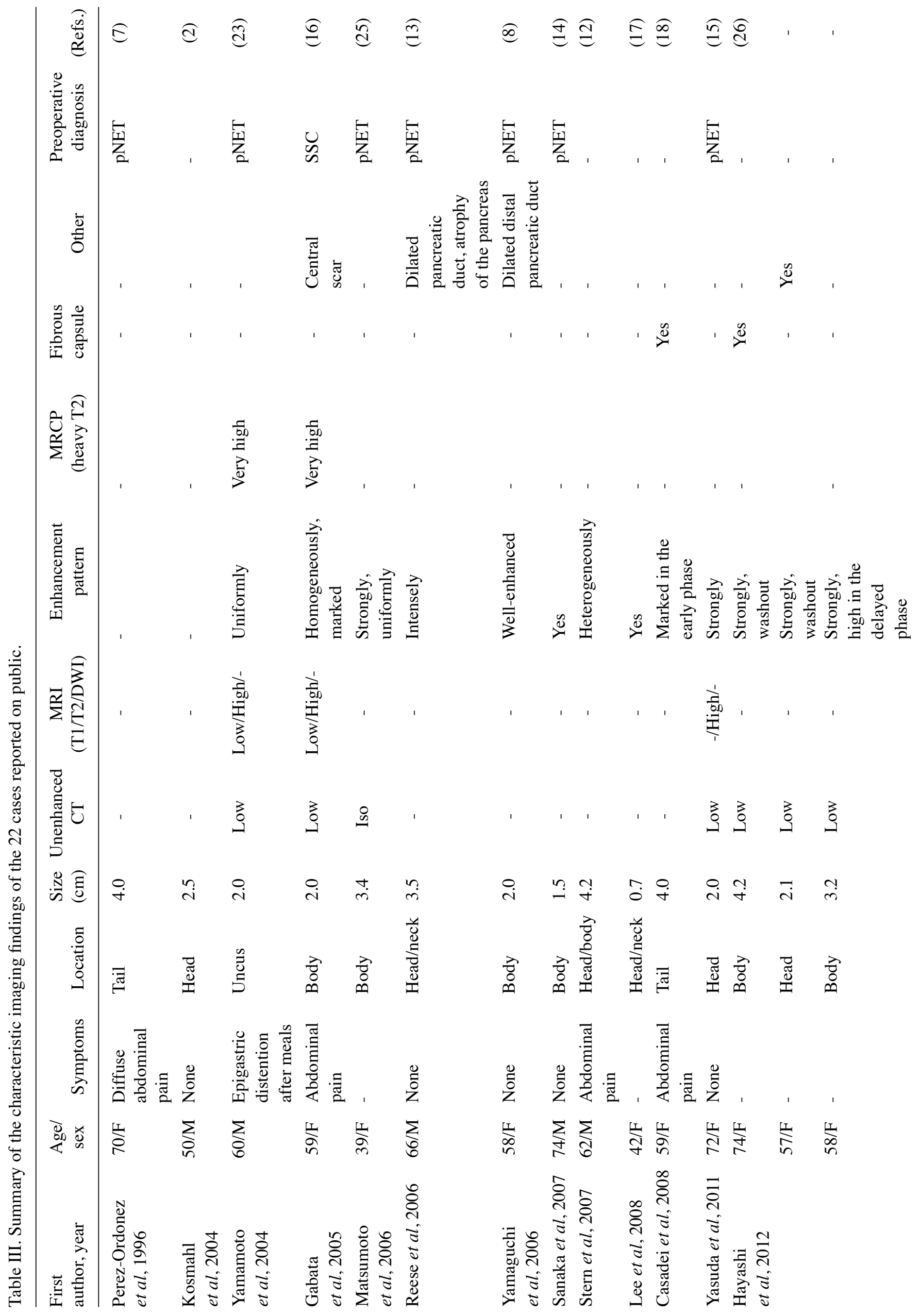




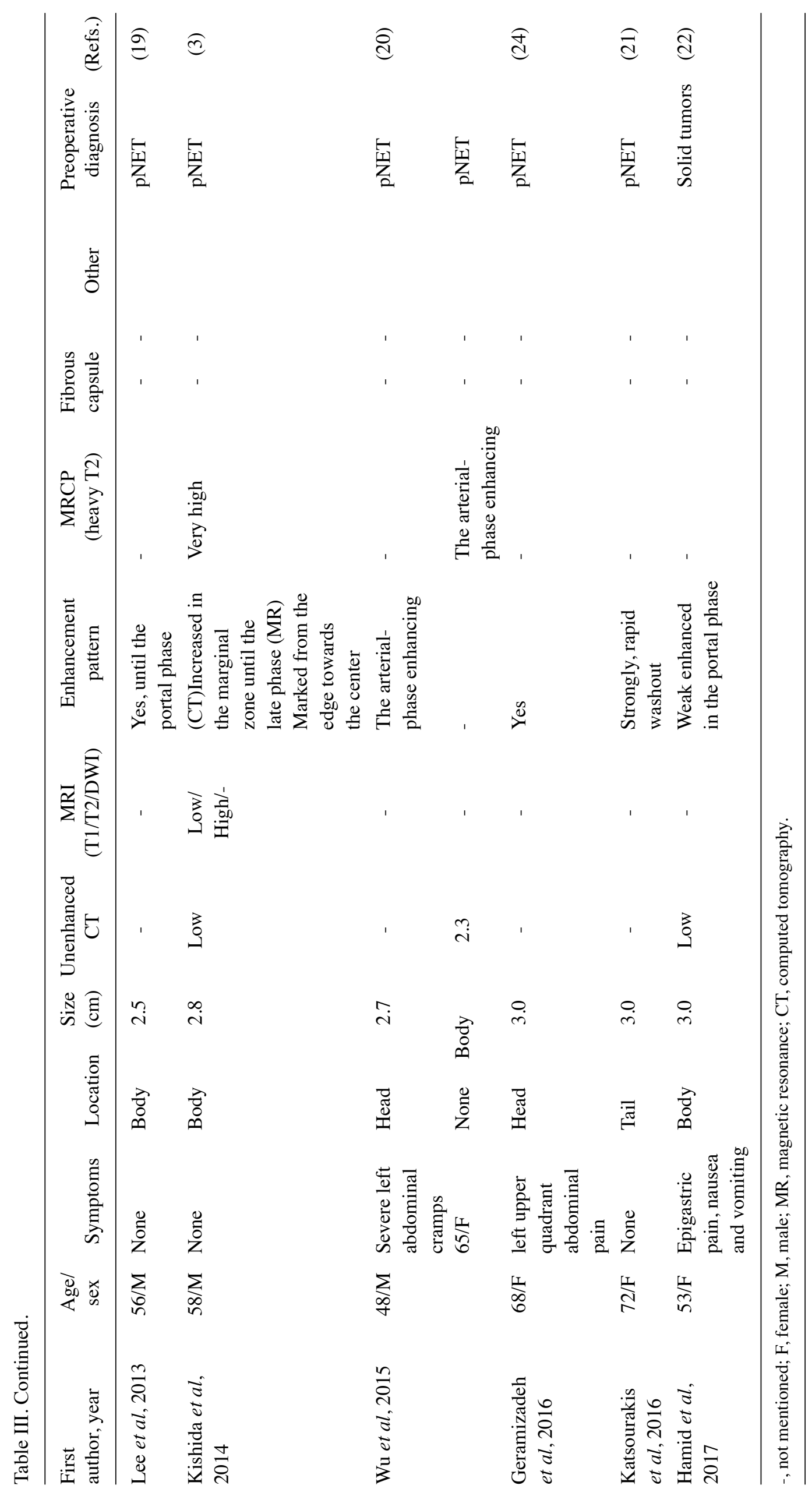


The SSCs in the present study were characterized as well-defined solid tumors with the following features: i) Small tumor size $(\leq 3 \mathrm{~cm})$; ii) significant high intensity on T2-weighted images; iii) wash-in and wash-out enhancement or moderate and prolonged enhancement; iv) high ADC value; and v) the presence of a fibrous capsule. The tumors demonstrated marked hyperintensity on T2-weighted images, revealing a liquid component and suggesting they were not solid but rather of cystic nature (16). MR imaging, which is able to detect the presence of tumors with a cystic nature (16), may be superior to CT scanning in differentiating SSCs from solid tumors. This was supported by a previously published case report with an exact preoperative diagnosis of SSC (16). In the current study, 2 masses exhibited wash-in and wash-out, which was similar to the majority of cases in previous studies $(21,26)$, while 3 cases exhibited moderate, gradual and prolonged enhancement and spotted enhancement in the latter 2 phases, similar to a SSC case reported by Lee et al (17). The $80 \%$ marginal area was clearly observed to demonstrate strong enhancement, and this was reported in previously published studies $(16,18,26)$. The reasons for the different enhancement patterns remain unknown, however, the high density of microvessels at the tumor margin and the presence of a surrounding fibrous capsule may be a contributing factor (3). It is possible that the degree of enhancement is positively associated with the amount of hypervascular stroma. The lesions had sharp margins and manifested no invasion of adjacent vessels or organs. The ADC values of the 5 lesions were all $>2 \times 10^{-3} \mathrm{~mm}^{2} / \mathrm{sec}$ and within the range of $2.06 \times 10^{-3}$ to $2.86 \times 10^{-3} \mathrm{~mm}^{2} / \mathrm{sec}$ as reported by Jang et al (27). Furthermore, this compares to an optimal ADC cut-off value of $1.21 \times 10^{-3} \mathrm{~mm}^{2} / \mathrm{sec}$ reported by Hayano et al that distinguished pancreatic cancer from noncancerous tissue (28), and $1.99 \times 10^{-3} \mathrm{~mm}^{2} / \mathrm{sec}$ that distinguished SSCs from pNETs (27). The ADC values in the current study exhibited no evidence of restricted diffusion, in accordance with their benign nature.

SSCs, particularly the smaller lesions, may have been difficult to distinguish from pNETs in the previous studies $(3,7,8,13-15,19-21,23-26)$ and SPNs in the current study. The majority of SSCs occur in females in the sixth decade of life, while pNETs may develop at any age, and small SPNs predominantly occur in females in their thirties and forties (11). SSCs with strongly or arterial-phase enhancement have been frequently misdiagnosed as pNETs $(3,8,15,20,21,25)$. Kishida et al (3) reported that MR images of pNETs exhibit a high intensity on the T2-weighted image, however, this was not as high as that of a cyst. However, hyperintensity on T2-weighted images may not be present in pNETs (27), particularly for small-sized lesions (29). A low radio density on unenhanced images and a tumor density $<32$ Hounsfield units on unenhanced CT images may aid in distinguishing them (27). SSCs frequently exhibit a reduced number of low density regions when compared with the surrounding pancreas parenchyma than pNETs (26). Hayashi et al (26) reported that the presence of a fibrous capsule may aid the differential diagnosis of SSCs from pNETs. Additionally, SSCs exhibit a lower density relative to the surrounding pancreas on the delayed phase CT more frequently than pNETs (26). This may explain the difference in the washout rate of contrast enhancement.
The gradual and prolonged enhancement was incorrectly diagnosed as SPN in the current study. Small $(\leq 3 \mathrm{~cm})$ SPNs demonstrate early heterogeneous and slightly progressive fill-in enhancement and seldom appear more enhanced than the normal pancreatic parenchyma (30). They are also initially hypoattenuating and slightly hypoattenuating or isoattenuating during the portal venous phase (31), which overlap with part of SSCs. Small SPNs have two important distinguishing features: The lack of the fibrous capsule $(11,30)$ and hyperintensity on DWI (32). In the present study, the majority of the SSCs displayed isointensity on DWI. Furthermore, the young age of the patients may have led to misdiagnosis of two cases in the current study. The tumor misdiagnosed as pancreatic adenocarcinoma with distal ductal dilation in the present study (case 4) may be distinguished using an increased signal intensity on T2-weighted image and high ADC value. SSCs only detected by unenhanced MR imaging and which appear as very hyperintense on T2-weighted images, mimicking the oligocystic type, may be misdiagnosed as unilocular mucinous cystic neoplasms and branch duct intraductal papillary mucinous neoplasms (BD-IPMNs). A thick cystic wall and curvilinear or peripheral calcification present in the cyst wall or septa occur in mucinous cystic neoplasms (33) and are important features for a differential diagnosis. BD-IPMNs are characterized by communication with the main duct which is apparent on MRCP (34).

The current study had two main limitations. Firstly, it was limited by its retrospective nature. Secondly, it included a small number of patients with pathologically proven SSCs that underwent surgical resection. This small number of patients was attributed to the rarity of the subtype.

In conclusion, SSCs were frequently observed in adult women with small $(\leq 3 \mathrm{~cm})$ lesions. Marked hyperintensity on T2-weighted images, a high ADC value, the presence of a fibrous capsule and enhancement patterns may well be imaging features of SSCs.

\section{Acknowledgements}

The authors would like to thank Dr Yuan-fei Lu and Dr Qian Zhang (The Second Affiliated Hospital of Zhejiang University School of Medicine, Zheijang, China) for making a significant contribution towards the revision of the manuscript.

\section{Funding}

No funding was received.

\section{Availability of data and materials}

All data generated or analyzed during this study are included in this published article.

\section{Authors' contributions}

RSY conceived the concept and designed the study. JYC and HYC collected cases and performed the study. YP and DS analysed the images, and prepared figures and tables. JYC wrote the paper. All authors read and approved the final manuscript. 


\section{Ethics approval and consent to participate}

The retrospective study was approved by The Second Affiliated Hospital of Zhejiang University School of Medicine Ethics Committee, and the requirement for obtaining informed consent from all patients was waived.

\section{Patient consent for publication}

Not applicable.

\section{Competing interests}

The authors declare that they have no competing interests.

\section{References}

1. Chandwani R and Allen PJ: Cystic neoplasms of the pancreas. Annu Rev Med 67: 45-57, 2016.

2. Kosmahl M, Wagner J, Peters K, Sipos B and Klöppel GN: Serous cystic neoplasms of the pancreas: An immunohistochemical analysis revealing alpha-inhibin, neuron-specific enolase, and MUC6 as new markers. Am J Surg Pathol 28: 339-346, 2004.

3. Kishida Y, Matsubayashi H, Okamura Y, Uesaka K, Sasaki K, Sawai H, Imai K and Ono H: A case of solid-type serous cystadenoma mimicking neuroendocrine tumor of the pancreas. J Dig Dis 15: 211-215, 2014.

4. Sun HY, Kim SH, Kim MA, Lee JY, Han JK and Choi BI: CT imaging spectrum of pancreatic serous tumors: Based on new pathologic classification. Eur J Radiol 75: e45-e55, 2010.

5. Kimura W, Moriya T, Hirai I, Hanada K, Abe H, Yanagisawa A, Fukushima N, Ohike N, Shimizu M, Hatori T, et al: Multicenter study of serous cystic neoplasm of the Japan pancreas society. Pancreas 41: 380-387, 2012.

6. Machado MC and Machado MA: Solid serous adenoma of the pancreas: An uncommon but important entity. Eur J Surg Oncol 34: 730-733, 2008 .

7. Perez-Ordonez B, Naseem A, Lieberman PH and Klimstra DS: Solid serous adenoma of the pancreas. The solid variant of serous cystadenoma? Am J Surg Pathol 20: 1401-1405, 1996.

8. Yamaguchi M: Solid serous adenoma of the pancreas: A solid variant of serous cystadenoma or a separate disease entity? J Gastroenterol 41: 177-178, 2006.

9. European Study Group on Cystic Tumours of the Pancreas: European evidence-based guidelines on pancreatic cystic neoplasms. Gut 67: 789-804, 2018.

10. Jais B, Rebours V, Malleo G, Salvia R, Fontana M, Maggino L, Bassi C, Manfredi R, Moran R, Lennon AM, et al: Serous cystic neoplasm of the pancreas: A multinational study of 2622 patients under the auspices of the International Association of Pancreatology and European Pancreatic Club (European Study Group on Cystic Tumors of the Pancreas). Gut 65: 305-312, 2016.

11. Yu MH, Lee JY, Kim MA, Kim SH, Lee JM, Han JK and Choi BI: MR imaging features of small solid pseudopapillary tumors: Retrospective differentiation from other small solid pancreatic tumors. AJR Am J Roentgenol 195: 1324-1332, 2010.

12. Stern JR, Frankel WL, Ellison EC and Bloomston M: Solid serous microcystic adenoma of the pancreas. World J Surg Oncol 5: 26, 2007.

13. Reese SA, Traverso LW, Jacobs TW and Longnecker DS: Solid serous adenoma of the pancreas: A rare variant within the family of pancreatic serous cystic neoplasms. Pancreas 33: 96-99, 2006.

14. Sanaka MR, Kowalski TE, Brotz C, Yeo CJ, McCue P and Palazzo J: Solid serous adenoma of the pancreas: A rare form of serous cystadenoma. Dig Dis Sci 52: 3154-3156, 2007.

15. Yasuda A, Sawai H, Ochi N, Matsuo Y, Okada Y and Takeyama H: Solid variant of serous cystadenoma of the pancreas. Arch Med Sci 7: 353-355, 2011.

16. Gabata T, Terayama N, Yamashiro M, Takamatsu S, Yoshida K, Matsui O, Usukura M, Takeshita M and Minato H: Solid serous cystadenoma of the pancreas: MR imaging with pathologic correlation. Abdom Imaging 30: 605-609, 2005.
17. Lee SE, Kwon Y, Jang JY, Kim YH, Hwang DW, Kim MA, Kim SH and Kim SW: The morphological classification of a serous cystic tumor (SCT) of the pancreas and evaluation of the preoperative diagnostic accuracy of computed tomography. Ann Surg Oncol 15: 2089-2095, 2008

18. Casadei R, D'Ambra M, Pezzilli R, Ricci C, Calculli L, Lega S, Antonacci N, Monari F and Minni F: Solid serous microcystic tumor of the pancreas. JOP 9: 538-540, 2008.

19. Lee SD, Han SS and Hong EK: Solid serous cystic neoplasm of the pancreas with invasive growth. J Hepatobiliary Pancreat Sci 20: 454-456, 2013.

20. Wu W, Hong X, Li J, Dai M, Wang W, Tong A, Zhu Z, Dai H and Zhao Y: Solid serous cystadenoma of the pancreas: A case report of 2 patients revealing vimentin, $\beta$-catenin, $\alpha-1$ antitrypsin, and $\alpha-1$ antichymotrypsin as new immunohistochemistry staining markers. Medicine (Baltimore) 94: e644, 2015.

21. Katsourakis A, Dimitriou I, Noussios G, Chatzis I and Chatzitheoclitos E: Solid serous adenoma of the pancreas: A case report and review of the literature. Case Rep Surg 2016: 3730249, 2016.

22. Hamid M, Tbouda M, Majbar AM, Raiss M and Ahallat M: Pancreatic solid serous cystadenoma treated by laparoscopy: Presentation of a new case report and review of the literature. Int J Surg Case Rep 40: 97-101, 2017.

23. Yamamoto T, Takahashi N, Yamaguchi T and Imamura Y: A case of solid variant type of pancreatic serous cystadenoma mimicking islet cell tumor. Clin Imaging 28: 49-51, 2004.

24. Geramizadeh B, Dabbaghmanesh $\mathrm{MH}$, Nikeghbalian S and Soleimani N: Solid serous adenoma of pancreas, misdiagnosed as neuroendocrine tumor, a rare case report and review of the literature. J Gastrointest Cancer 47: 462-465, 2016.

25. Matsumoto M, Iguchi M, Ohtsuki Y, Kimura M, Watanabe R, Watanabe N, Okada Y, Kurabayashi A, Takahashi T and Furihata M: A case of solid serous adenoma of the pancreas ultrastructurally harbouring ribosome-lamella complexes. Pathology 38: 361-364, 2006.

26. Hayashi K, Fujimitsu R, Ida M, Sakamoto K, Higashihara H, Hamada Y and Yoshimitsu K: CT differentiation of solid serous cystadenoma vs endocrine tumor of the pancreas. Eur J Radiol 81: e203-e208, 2012

27. Jang KM, Kim SH, Song KD, Kim YK, Lee SJ and Choi D: Differentiation of solid-type serous cystic neoplasm from neuroendocrine tumour in the pancreas: Value of abdominal MRI with diffusion-weighted imaging in comparison with MDCT. Clin Radiol 70: 153-160, 2015.

28. Hayano K, Miura F, Amano H, Toyota N, Wada K, Kato K, Sano K, Takeshita K, Aoyagi T, Shuto K, et al: Correlation of apparent diffusion coefficient measured by diffusion-weighted MRI and clinicopathologic features in pancreatic cancer patients. J Hepatobiliary Pancreat Sci 20: 243-248, 2013.

29. Bakir B, Salmaslioğlu A, Poyanlı A, Rozanes I and Acunas B: Diffusion weighted MR imaging of pancreatic islet cell tumors. Eur J Radiol 74: 214-220, 2010.

30. Yao X, Ji Y, Zeng M, Rao S and Yang B: Solid pseudopapillary tumor of the pancreas: Cross-sectional imaging and pathologic correlation. Pancreas 39: 486-491, 2010.

31. Baek JH, Lee JM, Kim SH, Kim SJ, Kim SH, Lee JY, Han JK and Choi BI: Small $(<\mathrm{or}=3 \mathrm{~cm})$ solid pseudopapillary tumors of the pancreas at multiphasic multidetector CT. Radiology 257: 97-106, 2010.

32. Jang KM, Kim SH, Kim YK, Park MJ, Lee MH, Hwang J and Rhim H: Imaging features of small $(\leq 3 \mathrm{~cm})$ pancreatic solid tumors on gadoxetic-acid-enhanced MR imaging and diffusion-weighted imaging: An initial experience. Magn Reson Imaging 30: 916-925, 2012.

33. Javadi S, Menias CO, Korivi BR, Shaaban AM, Patnana M, Alhalabi K and Elsayes KM: Pancreatic calcifications and calcified pancreatic masses: Pattern recognition approach on CT. AJR Am J Roentgenol 209: 77-87, 2017.

34. Sahani DV, Kadavigere R, Blake M, Fernandez-Del Castillo C, Lauwers GY and Hahn PF: Intraductal papillary mucinous neoplasm of pancreas: Multi-detector row CT with $2 \mathrm{D}$ curved reformations-correlation with MRCP. Radiology 238: 560-569, 2006.

This work is licensed under a Creative Commons Attribution-NonCommercial-NoDerivatives 4.0 International (CC BY-NC-ND 4.0) License. 